\title{
A methodology for equitable performance assessment and presentation of wave energy converters based on sea trials
}

\author{
J.P. Kofoed ${ }^{a}$, A. Pecher ${ }^{\text {a,* }}$, L. Margheritini ${ }^{a}$, M. Antonishen ${ }^{\text {a }}$, C. Bittencourt ${ }^{b}$, B. Holmes ${ }^{c}$, C. Retzler $^{c}$, \\ K. Berthelsen ${ }^{d}$, I. Le Crom ${ }^{e}$, F. Neumann ${ }^{e}$, C. Johnstone ${ }^{f}$, T. McCombes ${ }^{f}$, L.E. Myers ${ }^{g}$ \\ a Aalborg University, Department of Civil Engineering, Sohngaardsholmsvej 57, 9000 Aalborg, Denmark \\ ${ }^{\mathrm{b}}$ Det Norske Veritas BV, The Netherlands \\ ${ }^{\mathrm{c}}$ University College Cork, Ireland \\ ${ }^{\mathrm{d}}$ Aalborg University, Denmark \\ ${ }^{\mathrm{e}}$ Wave Energy Centre, Portugal \\ ${ }^{\mathrm{f}}$ University of Strathclyde, UK \\ ${ }^{\mathrm{g}}$ University of Southampton, UK
}

\section{A R T I C L E I N F O}

\section{Article history:}

Received 14 June 2011

Accepted 28 October 2012

Available online 22 November 2012

\section{Keywords:}

Wave energy converters

Performance assessment

Annual energy production

Wave to wire

Sea trials

\begin{abstract}
A B S T R A C T
A general and widely applicable methodology to assess and present the performance of wave energy converters (WEC) based on sea trials is presented. It is meant to encourage WEC developers to present the performance of their WEC prototypes, on a transparent and equitable way while taking care of possible discrepancy in the observed performance of the WEC. Due to the harsh uncontrollable conditions of the sea that is encountered by WECs during sea trials, some of the performance of the WECs might be sub optimal and the data sets not fully complete. The methodology enables to filter the data by applying a selection criterion on the performance data that was obtained for a certain range of wave conditions. This selection criteria result in a subset of performance data representing the performance of the WEC for specific wave conditions, from which an average value an appreciation of the related uncertainty can be derived. This can lead to the estimation of the annual energy output of the WEC at its test location, while it also provides a method to estimate its annual energy output for another location of interest and possibly also at another scaling ratio. The same methodology can also be used to perform parametric studies with environmental or device dependent parameters and to analyse the power conversion chain from wave to wire, which both could lead to an enhanced understanding of the performance and behaviour of the WEC.

The same methodology is also applicable to tidal devices or any other developing technologies that are used in an uncontrollable environment.
\end{abstract}

(c) 2012 Elsevier Ltd. All rights reserved.

\section{Introduction}

Numerous documents have been published by different research entities in various countries regarding the development and performance analysis of wave energy converters (WEC) based

\footnotetext{
* Corresponding author. Tel.: +45 994084 74; fax: +45 99408552 .

E-mail addresses: jpk@civil.aau.dk (J.P. Kofoed), afsp@civil.aau.dk (A. Pecher), lm@civil.aau.dk (L. Margheritini), michael.antonishen@gmail.com (M. Antonishen), Claudio.Bittencourt.Ferreira@dnv.com (C. Bittencourt), B.Holmes@ucc.ie (B. Holmes), c.retzler@pelamiswave.com (C. Retzler), kkb@ math.aau.dk (K. Berthelsen), izan@wave-energy-centre.org (I. Le Crom), frank@ wave-energy-centre.org (F. Neumann), cameron.johnstone@strath.ac.uk (C. Johnstone), tom.mccombes@strath.ac.uk (T. McCombes), l.e.myers@soton.ac.uk (L.E. Myers).
}

on tank testing and sea trials. However, it has been shown that there is a need of having even more developed standards in ocean energy as standards are expected to contribute positively to the development of the industry [1,2]. Existing literature on the presentation and analysis of WEC sea trial performance data focuses primarily on commercially ready or well-established devices rather than those under development. Some of these related documents are [3-7].

This paper provides a methodology for the analysis and presentation of data obtained from sea trials of wave energy converters and is an expansion of the related EquiMar protocols [8]. The equitable aspect of this methodology lies in its wide application, as any WEC at any scale or stage of development can be considered as long as the tests are performed in real sea conditions, and that the results contain statistical information concerning the 


\begin{tabular}{|c|c|c|c|}
\hline \multicolumn{2}{|c|}{ Nomenclature } & $P_{\text {average }}$ & average power output [W] \\
\hline AEP & annual energy production [Wh] & $s$ & sample standard deviation \\
\hline $\begin{array}{l}\text { Contrib } \\
\mathrm{CI}\end{array}$ & $\begin{array}{l}\text { contribution to the available wave energy resource [-] } \\
\text { confidence interval }\end{array}$ & $t^{*}$ & $\begin{array}{l}\text { statistical parameter representing the confidence level } \\
\text { of the confidence interval }\end{array}$ \\
\hline CL & confidence level & $T_{e}$ & wave energy period $\left(m_{-1} / m_{0}\right)[s]$ \\
\hline$H_{\mathrm{mo}}$ & estimate of the significant wave height derived from & $v$ & characteristic velocity $[\mathrm{m} / \mathrm{s}]$ \\
\hline$g$ & $\begin{array}{l}\text { spectral moment, } 4 \sqrt{ } m_{0}[\mathrm{~m}] \\
\text { gravitational acceleration }\left[\mathrm{m} / \mathrm{s}^{2}\right]\end{array}$ & $\eta$ & $\begin{array}{l}\text { non-dimensional power performance (also referred to } \\
\text { as efficiency) [-] }\end{array}$ \\
\hline $\begin{array}{l}n \\
m_{n}\end{array}$ & $\begin{array}{l}\text { number of data points } \\
\text { spectral moment of the nth order }\left[\mathrm{m}^{2} \mathrm{f}^{\mathrm{n}}\right]\end{array}$ & $\eta_{\text {zone }}$ & $\begin{array}{l}\text { non-dimensional power performance for the wave } \\
\text { conditions of a zone [-] }\end{array}$ \\
\hline $\begin{array}{l}L \\
\mathrm{LF}\end{array}$ & dimension, e.g. length $[\mathrm{m}]$ & $\eta_{i}$ & non-dimensional power performance of \\
\hline$P$ & power output [W] & $\lambda$ & scaling ratio, requiring geometrical similarity $=L_{f} / L_{m}[-]$ \\
\hline Prob & probability of occurrence [-] & WEC & wave energy converter \\
\hline
\end{tabular}

stated performance of the WEC. This will allow the estimation of the annual energy production (AEP) of the WEC at any scaling ratio and location of interest. The representation of the performance will be better resolved when more performance data has been gathered and consequently its statistical reliability will be increased.

The harsh environmental conditions in which sea trials are performed involve a large range of engineering development and device monitoring challenges, since the offshore environment is by nature uncontrollable and only predictable to a certain extent. Unfortunately this can incur significant costs during device development and, even with heavy investment, some WEC components or measurements can still perform poorly. In addition, having the WEC tested in every possible sea state can require very long trials, since some conditions occur only very infrequently. This often leads to testing campaigns that are not as extensive as desired. Therefore, the performance analysis should be robust enough to allow using suboptimal performance data that are obtained from sea trials that are not fully completed. In other words, this methodology is focused at retrieving the maximum amount of useful information out of incomplete data sets.

This methodology presents means to assess the performance of a WEC, tested in real sea conditions, by evaluating its performance (in a first approach) separately for different wave conditions. These "different wave conditions" are defined as zones and the range of their corresponding wave parameters is defined in accordance with the availability of data and resolution of the scatter diagram [9]. For each of these zones, the performance of the WEC will be stated together with a statistical parameter describing the reliability of the stated performance. Based on the performance of each zone, an overall appraisal of the performance can then be created. Once the non-dimensional performance is characterised for the specific wave parameters of each zone, it can be translated to scatter diagrams of other locations of interest or used for different scales of the device. The methodology facilitates as well parametric studies, as the performance data can be chosen accordingly.

The uncertainty of the performance for a zone is based on the selected performance data that are chosen to represent the sea conditions. A minimum number of data points must be considered for each range of zones in order to ensure that the results are both repeatable and reproducible, and a limit is set regarding the maximum range of a sea state. Although the representation of the environmental conditions in which the tests occur will be reduced to the bi-variate $H_{m 0}-T_{e}$ scatter diagram, the influence of other environmental parameters is still expected to be present in the representation of the performance by the inclusion of different data points for each sea state. The extent to which other environmental parameters or even device dependent parameters influence the performance of the WEC can also be investigated using this methodology.

\section{Overview of the methodology}

A schematic overview of the different steps and possible applications of this methodology are given in Fig. 1. The arrows surrounding the figure indicate that the process can be repeated throughout the sea trials, especially when more data becomes available.

In general, while the same methodology can be used at any stage of development of a WEC as long as the tests are performed in real sea conditions, there are three principal applications:

- The assessment of the AEP of the WEC at its test location. The results of this analysis can subsequently be used to estimate the performance of the same WEC at another location of interest. In the case that the device is to be scaled or used at another location, the data can be scaled correspondingly and the results can be used to validate a numerical data model.

- The assessment of the available energy at various stages of the energy conversion chain from wave-to-wire. This offers an individual view on specific energy conversion steps, allowing characterisation of each steps behavioural trend relative to the wave conditions.

- The assessment of the influence of a specific environmental or device dependent parameter on the performance of the WEC, possibly at various energy conversion steps.

Irrespective of the eventual application of the method, the procedure consists of three main parts. The first part intends to preprocess the environmental data (e.g. waves, tides, wind, etc.) and the performance data (e.g. mechanical power, electricity transmitted to the grid, etc.). This consists of establishing the environmental matrix that contains all parameters used to characterise the environmental climate at the test site. This environmental matrix has to be based on long term data, typically 10 years or more for wave data [10], in order to cover all the long and short term variability of the individual parameters. This can then be simplified into the scatter diagram, determined by $H_{m 0}$ and $T_{e}$. The performance of the WEC at the conversion stage of interest - in terms of power output $(P)$ or available power - is processed relative to its corresponding environmental conditions in order to obtain the nondimensional performance values of the WEC, and forms the basis of the procedure. In cases where various energy conversion steps or other device dependent or environmental parameters are investigated, then they must also be included in the data at the beginning. 


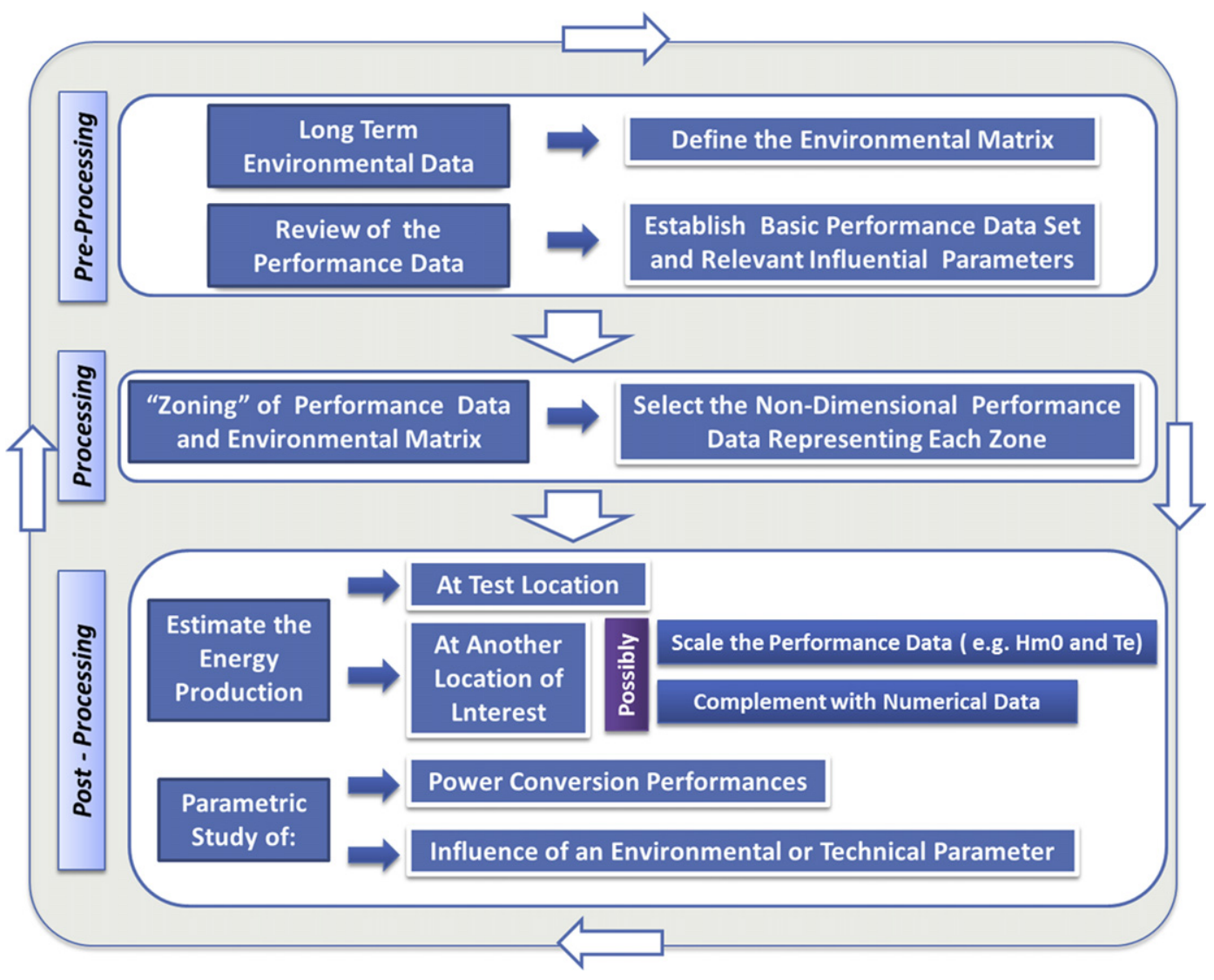

Fig. 1. Overview of the equitable performance assessment and presentation methodology.

As they will impact the later development of the process, it is necessary to capture their impact on the initial data.

The processing of the environmental and performance data is achieved by clustering the data into "zones." Each zone is delimited by a specific range of the environmental matrix (typically in terms of $H_{m 0}$ and $T_{e}$ ) and includes a certain amount of performance data points. For each zone, a non-dimensional power performance ( $\left.\eta_{\text {zone }}\right)$ will be calculated, together with a corresponding uncertainty based on a selected subset of the performance data points that are included in the zone. The size and the location of the zones on the environmental matrix are defined corresponding to the available data and overall environmental matrix. The selection of the data points that represent a zone has to be done carefully as it influences the stated $\eta_{\text {zone }}$ and its related uncertainty, which are at the basis of the calculation of the AEP of the WEC.

The post-processing summarises and presents the outcomes of the specific application of the methodology, as identified at the beginning of this section.

\section{Pre-processing of the data}

\subsection{Environmental matrix}

Characterising the real sea environment for which WECs are intended is complex, as the number of parameters that are involved is large and their possible definition can be ambiguous, e.g. the description of the characteristic wave height. The environmental matrix, comprising $n$ dimensions, therefore represents a summary of the numerous parameters describing the environmental conditions at a particular location. To capture the overall variability of the environment, they should be based on time series of data covering sufficient duration - 10 years or more for waves [10] - although it can be difficult to find 10 years of verified measured wave data for a particular site. Therefore, in order to maintain the minimal environmental uncertainty, it is common to resort to computer model predicted data, using a validated model, typically calibrated against short-term (3-4 months) measurements at location. Some of the main required environmental parameters used with wave energy converters are:

- $H_{m 0}$ :Significant wave height derived from spectral moments, $4 \sqrt{ } m_{0}$

- $T_{e}$ :Energy period $\left(m_{-1} / m_{0}\right)$

- Groupiness factor and wave height distribution

- Spectral characteristics (e.g. spectral width, etc.)

- Dominant wave direction and directional spreading

- Tidal range

- Water current speed and direction

In order to condense the large amount of information in the ndimensional environmental matrix and to make it easier to use, it has to be simplified. This results in a scatter diagram that summarises the long term average probabilities of occurrence (Prob) of all wave height-wave period combinations $\left(H_{m 0}, T_{e}\right)$. The environmental scatter diagram is segmented into "bins", of typically $0.5 \mathrm{~m}$ of wave height by $1 \mathrm{~s}$ of wave period. However, depending on the conditions (and accuracy of the environmental parameters etc.), the sizes of the bins can be adapted. For each bin, representing a defined sea state, the probability of occurrence has to be calculated.

In some cases, characterisation of the environment at the exact location of the WEC is not possible, but is at some distance around 
it. For deep water conditions, this does not have a significant impact, but for WECs placed on or at the coast line or in shallow water sites, the measurements might not adequately represent the conditions at the location of the WEC, e.g. the measured parameters can be influenced by the object interference or diffraction, including from the WEC itself. In this case, advanced 3D wave measurement is required or another distant but robust measurement can be used as reference for the plant, e.g. in deep water conditions. The distant wave measurement might not very accurately represent the wave conditions at the plant, but it is easier to implement and is more reproducible, and therefore it can also be more representative in order to estimate the AEP of the same WEC at another location of interest.

\subsection{Configuration of the data}

All the measured time series of environmental and performance data are reduced into characteristic values, representative over a defined time span. The most commonly used time span is half an hour [11], as for typical conditions it gives the best trade-off between the accurate evaluation of the observed environmental parameters and the statistical accuracy of the measurements. The data selection will exclusively be based on the non-dimensional device performance parameters and the environmental parameters; therefore the corresponding $\eta$ for every performance data point $\left(\eta_{i}\right)$ should be included from the beginning. The $\eta$ (sometimes also referred to as efficiency) is the ratio between the power output at the stage of consideration $P$, e.g. power supplied to the grid, and the available wave power resource $P_{\text {wave }}$ for the given characteristic width of the WEC (Eq. (1)).

$\eta_{i}=\frac{P}{P_{\text {wave }} \cdot \text { width }}$

Depending on the desired application, the amount of environmental, performance or device dependent parameters of interest can differ. In the basic case, where the performance of the WEC and the estimation of its power production capabilities at test or other location are assessed, only the most important wave parameters (normally $H_{m 0}, T_{e}$ and $P_{\text {wave }}$ ) and the power output is processed for every data point. The power output can be calculated at any stage of the conversion chain between the available wave power and the electrical power supplied to the grid. For full-scale devices it is desirable to do the power assessment as close to the grid connection as possible in order to obtain as confident power production estimates as possible. For small scale devices it can be useful to use an earlier reference in the power conversion chain, from wave to wire, as losses in various power conversion steps might change with device scale and power conversion losses typically do not follow the Froude scaling law, which is typically used for the scaling environmental parameters and overall geometry of the WEC. These losses must be scaled following their specific scaling law and thus still be integrated.

If the performance of the WEC is to be assessed at various power conversion stages, then the related non-dimensional performance parameters should be included. However, in the case that the influence of another parameter, environmental or device dependent is assessed, then the data should be arranged relative to this new parameter. In other words, the data should be separated into different data sets with respect to the influential parameter that is being analysed. This influential parameter of interest can be of any kind, e.g. it could define an aspect of the waves, such as their groupiness factor, or it could define an aspect of the WEC, e.g. a control strategy or some configuration of the WEC or one of its components.

\section{Processing of the data}

\subsection{Zoning}

The definition of the zoning, which divides the $H_{m 0}-T_{e}$ scatter diagram in various areas or "zones", should be done according to the available performance data and the environmental scatter diagram. A zone should not be larger than necessary, and can even be resolved down to the size of a bin of the environmental scatter diagram, but it should contain sufficient data points. The number of data points is important in order to obtain an average $\eta$ of the selected performance data in a zone $\left(\eta_{\text {zone }}\right)$ at an acceptable statistical uncertainty (explained more in detail in Section 4.2). It is also suggested to limit the size of a zone in such a way that a maximum of 20 per cent of the total available wave energy resource at the test location is found within a single zone. This will be referred to as the contribution to the available wave energy (Contrib, Eq. (2)). Whenever a zone includes various bins, it is recommended to enlarge the zone with respect to the least influential environmental parameter $\left(H_{m 0}\right.$ or $\left.T_{e}\right)$ on the performance of the device. An example of such enlarged zones is given in Fig. 2,
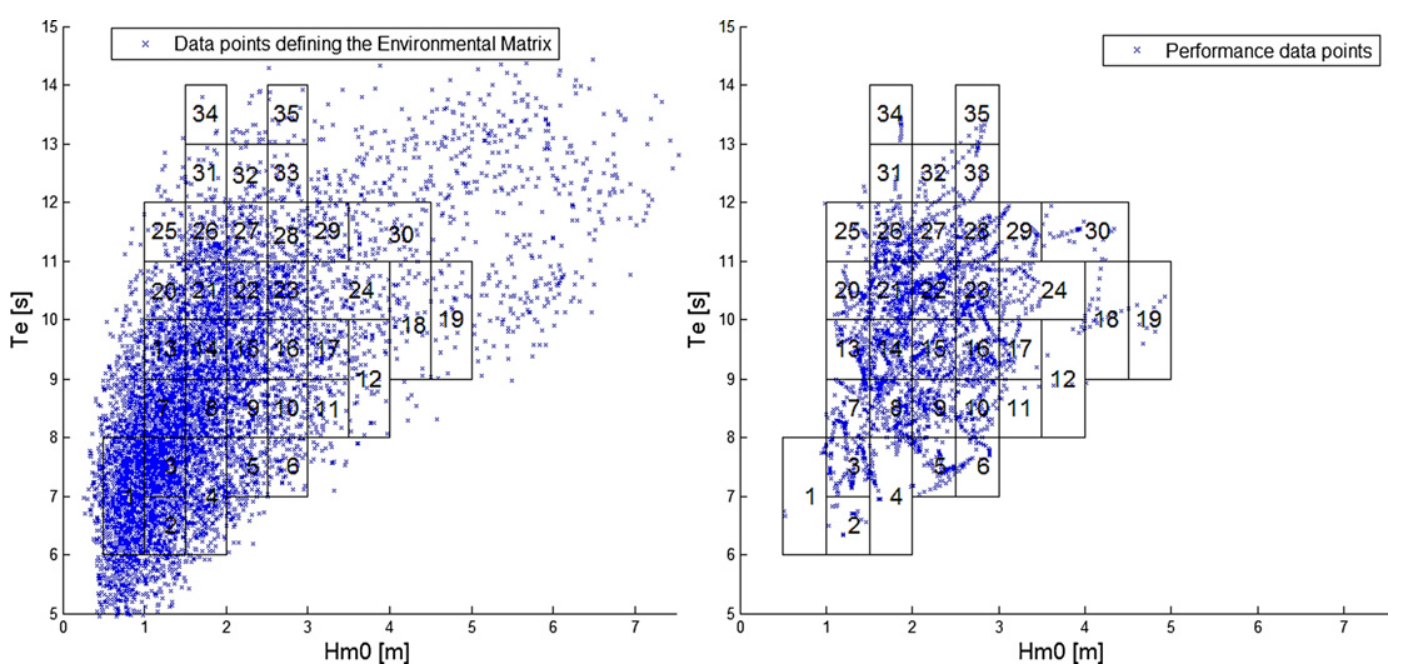

Fig. 2. The data points defining the environmental matrix (left) and the available performance data points (right), both with the zones overlaid. 
where zoning favours a good resolution on the wave height axis rather than the wave period axis, as the performance of the concerning WEC is less dependent on the wave period as on the wave height. The configuration of the zones can be chosen in order to present the best results and it should be re-evaluated over time when more performance data becomes available.

Fig. 2 presents examples of zone layouts over the environmental and the performance data. The zones are designed with a focus on representing the performance data, which can leave a part of the environmental scatter diagram uncovered. This could result from incomplete test procedures if these wave conditions did not occur during the sea trials, or because the WEC just does not operate in these wave conditions. In the first case, these unmarked zones can still be included in the AEP; however their performance will have to be based on numerical models. In the second case, the performance of the WEC will have to be set to zero for all the wave conditions in which it will not operate.

Every bin of the environmental scatter diagram has a characterising $H_{m 0}$ and $T_{e}$ value that corresponds to its centre and an associated probability of occurrence. This allows derivation of the corresponding wave power $\left(P_{\text {wave }}\right)$ - which should take the water depth and other environmental parameters into account - and the wave energy contribution (Contrib) to the overall wave energy resource. The wave energy contribution of every bin to the overall wave energy resource can be calculated by:

Contrib $_{\text {bin }}=\frac{\left(P_{\text {wave }}\right)_{\text {bin }} \cdot \text { Prob }_{\text {bin }}}{\sum_{\text {bin }=1}^{n}\left(\left(P_{\text {wave }}\right)_{\text {bin }} \cdot \text { Prob }_{\text {bin }}\right)}$

The characterising environmental parameters of every zone $\left(H_{m 0}\right.$ and $T_{e}$ ) are the average of the environmental parameters of the various bins included in the zone, weighted by their respective wave energy contribution. The corresponding equations to calculate the characterising $H_{s}$ and $T_{e}$ for each sea state are:

$H_{m 0 \text { zone }}=\sqrt{\frac{\sum_{\text {bin }=1}^{n} H_{m 0 \text { bin }}^{2} \cdot \text { Prob }_{\text {bin }}}{\sum_{\text {bin }=1}^{n} \text { Prob }_{\text {bin }}}}$

and

$T_{\text {ezone }}=\frac{\sum_{\text {bin }=1}^{n} T_{e \text { bin }} \cdot \text { Prob }_{\text {bin }}}{\sum_{\text {bin }=1}^{n} \text { Prob }_{\text {bin }}}$

The probability of occurrence (Prob ${ }_{\text {zone }}$ ) and the wave energy contribution (Contrib zone $_{\text {) }}$ of a zone is the sum of the respective values of the bins that are included in each.

\subsection{Selection of the performance data}

A central element in the method is that a subset of all the performance data points for each zone is selected, and this subset is then used for the calculation of the non-dimensional performance $(\eta)$ and related statistical uncertainty of the performance of the WEC for the environmental conditions represented by the zone.

The selection of the performance data points should be done carefully, observing the following stipulations:

- The performance of the WEC must be supplied in terms of the non-dimensional performance $(\eta)$, as the selection of performance data points has to be done on their respective $\eta$ and not on their absolute performance in $\mathrm{kW}$. For full-scale devices it is suggested to use the final power measurement as reference, while for device in reduced scale the first power measurement on the device should be used (Section 3.2).
- It is recommended to include at least 5 performance data points in the selection of every zone. However, it is strongly encouraged to increase this number in order to present more robust values and the amount of selected performance data does not need to be the same in each zone.

- The selection of which data points to include is not strictly specified, since it is a question of compromise. Including more data points into the subset will typically result in lowering the corresponding statistical uncertainty but also the corresponding $\eta_{\text {zone }}$ and vice-versa. Therefore, the selection of data for the subset consists of finding the right balance between the stated $\eta_{\text {zone, the related statistical uncertainty and the quantity of }}$ performance data points to include.

- The fact that only a subset from all the available performance data is used indicates that the WEC is still in its development process; otherwise all the acquired performance data should be used to represent the overall performance. It is obvious that performance data points having especially low performances will not be favourable for inclusion in the subset. Similarly, performance data presenting anomalous high performances can be problematic, since they would increase the uncertainty on the stated $\eta_{\text {zone }}$ considerably. These data points should also be excluded as they probably result from either inaccurate wave measurements or some occasional beneficial event. Such an example can be seen in zone 2 and 3 on the left of Fig. 3, where some high performance data points are not included in the selection.

- Consistent selection criteria must be used when choosing the performance data points in all the zones, and should be stated with the results. This would enable the reproducibility of the results of this methodology.

There is at present no commonly accepted, widely applicable or optimal selection criterion for performance data points and as such none is specified herein. Developments of suitable criteria are anticipated to come through an extensive use of this methodology on different types of WEC in different development phases and are likely to be fairly project specific: the various foreseeable selection criteria might possibly be WEC, instrumentation, location or case dependent. Two applied case studies can be found in [12] and [13].

In Fig. 3, the selection of the performance data is presented for each zone in two different ways. The left plot presents $\eta$ for the selected data points (larger red dots) and of the non-selected performance data (smaller green dots) for each zone, together with $\eta_{\text {zone }}$ and the related confidence interval (indicated by the larger circle and triangles). The plot on the right presents the selected performance data (larger red dots) and the non-selected performance data (smaller green dots) together with the layout of the zones.

The left plot of Fig. 3, presents the selected and not-selected performance data points for each zone, together with the $\eta_{\text {zone }}$ and its related confidence interval. In almost all the zones, some performance data points with low performances have been excluded (green dots located below the $\eta_{\text {zone }}$ ), while in zones 2 and 3 data points with very high performances have been disregarded. This resulted from the selection criterion designed to avoid excessively large confidence intervals [12]. It could be argued that any other disproportionately high performance data points should be disregarded (e.g. in zone 5 or zone 20): this indicates again that the definition of the selection parameters is very subtle and difficult to generalise.

In the right plot in Fig. 3, the spreading of the selected and deselected performance data points can be seen over the zones. In most cases, the spreading of the selected performance data points is reasonably consistent (all the zones in the middle, with 

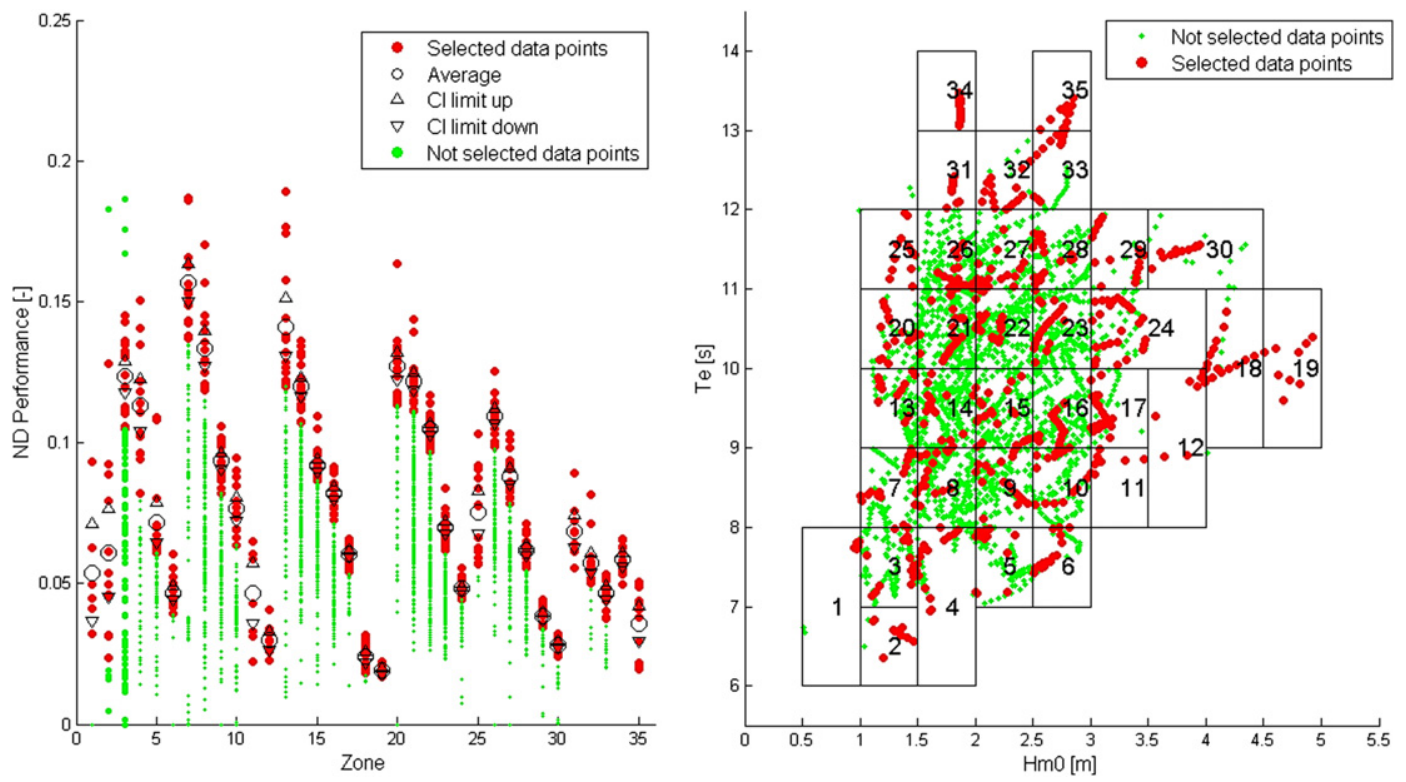

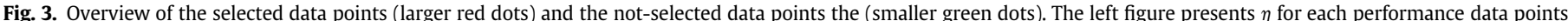

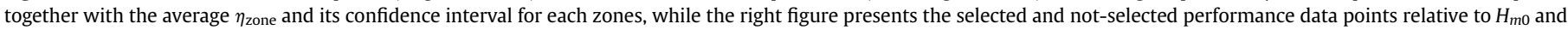
$T_{e}$. (For interpretation of the references to colour in this figure legend, the reader is referred to the web version of this article.)

a significant number of performance data points). In some other cases the spreading is limited (e.g. zone 1 and 11). These zones could have been disregarded, included in another zone or simply been allocated a zone (as it was the case here). This is also a very delicate choice and probably case dependent. In this particular case, the device was having structural problems that caused it (in general) not to be operational in small wave conditions; however in the next model these problems should be resolved. This makes the device operational in these conditions and thereby this zone ought to be included, as long as the performance data is good - which is the case - and can be seen in Fig. 3 (plot left). Another issue can arise from temporal correlation, which corresponds to selected performance data points that appear to be to consecutive measurements (zone 18 and 34). Although these performance data points are correct, they only represent a single event and thereby do not include a wide range of variations in environmental parameters that could be found for these wave conditions. This is likely to result in underestimating the "real" standard deviation and confidence interval.

A good verification of the representativeness of the stated $\eta_{\text {zone }}$ can be done by converting all the non-dimensional values into the absolute power output (expressed in kW), as can be seen in Fig. 4 . This can be done by multiplying the stated $\eta_{\text {zone }}-$ calculated following Eq. (5) - by the corresponding available wave power. This can be calculated with the characterising environmental parameters of a zone using Eqs. (3) and (4). In some cases, the stated absolute power production can be unrealistically higher than the data points on which it was based, e.g. zone 6 in Fig. 4. The reason can be that the selected performance data points are not well spread over the zone and are clustered more at the top or bottom of the zone. This can induce a kind of levering effect, and may result in a mismatch between the average $P_{\text {wave }}$ of the selected performance
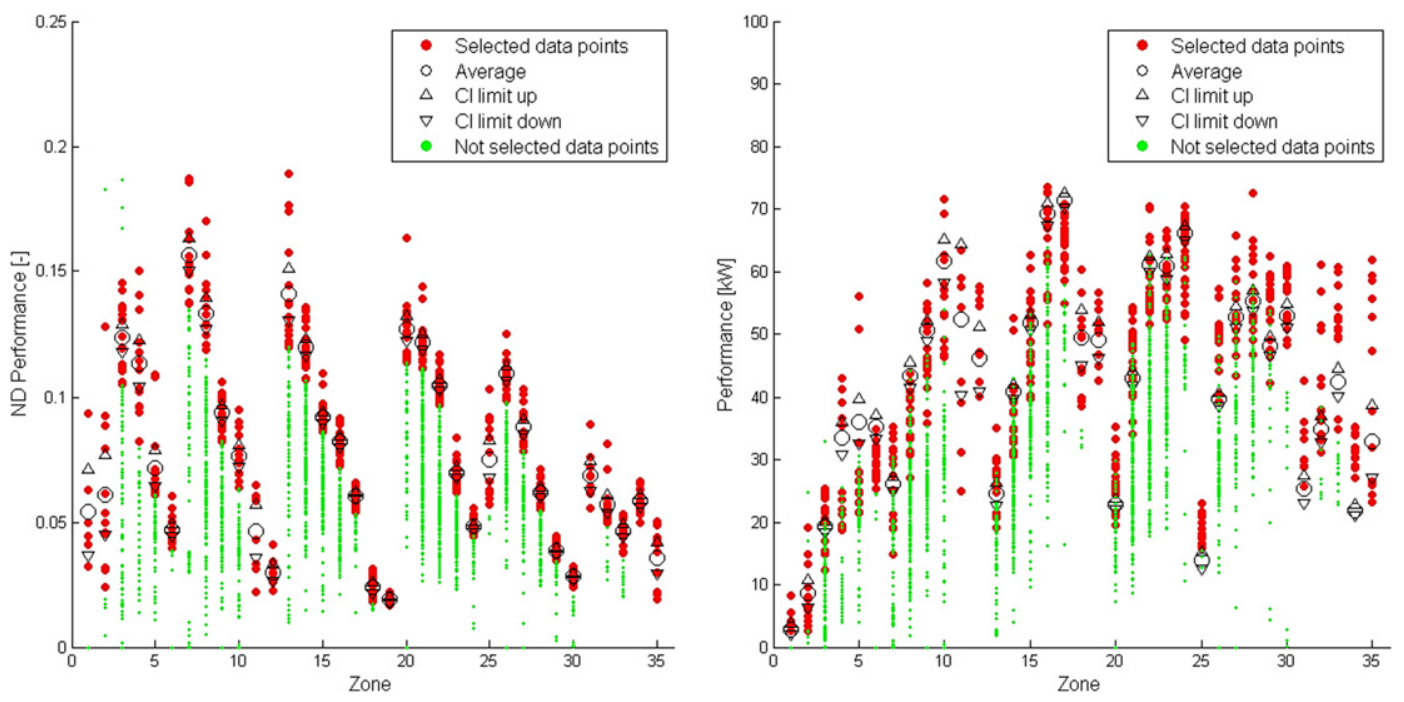

Fig. 4. Data selection set for each single zone for non-dimensional and absolute power production values. 
data and the of the $P_{\text {wave }}$ zone. The size or shape of the related zones can be modified in order to avoid this; however they are often restricted to the bins of the scatter diagram. Besides the selected and deselected performance data, Fig. 4 also presents the statistical uncertainty of the average value expressed as a confidence interval (Eq. (5)).

\subsection{Evaluation of the selected performance data}

The performance and the uncertainty parameters of each zone are calculated based on the selected data points. The $\eta_{\text {zone }}$ is calculated by taking the average of the non-dimensional performance $\left(\eta_{i}\right)$ of the selected data points in a zone (the left term in Eq. (5)). The confidence interval $\left(\mathrm{Cl}_{\text {zone }}\right)$ of the stated $\eta_{\text {zone }}$ can be calculated based on the level of confidence $\left(t^{*}\right)$, the sample standard deviation $(s)$ of the $\eta_{i}$ of the selected data points and the number of data points (n) which are considered. This provides an uncertainty margin

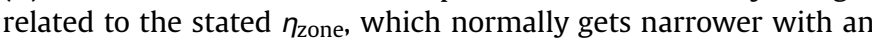
increasing number of data points or smaller standard deviation. The proposed confidence level is to be 95\% using the Student's t-distribution. In order to propose a general and common approach to describe the distribution of the $\eta$ of the performance data points in a zone, use of the Student's t-distribution is suggested. However, this distribution might not be the most suitable in all cases; especially as samples sizes can possibly be very small. In this case, more sophisticated treatment can be required, e.g. non-symmetric distributions, and the distribution used should clearly be stated with the performance assessment results.

This approach encourages the WEC operation to focus on demonstrating good performance over larger periods of time (resulting in a greater amount of performance data points) in order to stabilise the $\eta_{\text {zone }}$ and to reduce the $\mathrm{Cl}_{\text {zone. The average } \eta \text { and its }}$ corresponding confidence interval for each zone, based on the selected performance data points $(n)$, can be calculated as:

$\eta_{\text {zone }} \mp \mathrm{CI}_{\text {zone }}=\frac{\sum_{i=1}^{n} \eta_{i}}{n} \pm t * \frac{s}{\sqrt{n}}$

These non-dimensional values can be multiplied by the available $P_{\text {wave }}$ to obtain the absolute power output, and an overview table (Fig. 5) can be created stating the results for the main parameters of interest, in this case the zone number, $n, \eta_{\text {zone, }}, s_{\text {zone, }}$, Prob and $P_{\text {average }}$. Every zone also contains a blue dot, used for visual verification of its calculated "centre" in terms of $H_{\mathrm{mo}}$ and $T_{e}$, based on the wave energy contribution of its constituent bins in the scatter diagram.

Fig. 6 includes an overview of the distribution of the available wave power (left), an overview of the $\eta_{\text {zone }}$ (middle) spread over an $H_{m 0}-T_{e}$ diagram and the absolute power output $\left(P_{\text {average }}\right)$ on the right plot, which is based on the two previous plots. The plot on the right also corresponds to the effective power matrix, representing the performance of the WEC during sea trials.

\section{Post-processing and presentation of the data}

\subsection{Assessment of the performance and estimation of the energy production}

Based on the derived performance data for each zone, an overview can be created of the performance of the WEC (Fig. 6) as well as an estimation of its annual energy production (AEP). These values will indicate how the WEC has been performing, especially during the "better" periods, i.e. representing its potential and, as long as these conditions are maintained, how it can perform on an annual basis. The summarising table, given in Table 1, indicates the various parameters and results of each zone in three sections:

- The first section contains the environmental parameters such as the wave height $\left(H_{m 0}\right)$, wave period $\left(T_{e}\right)$, the available wave power $\left(P_{\text {wave }}\right)$, probability of occurrence (Prob), the multiplication of the two previous ( $P_{\text {wave }}{ }^{*}$ Prob) and its contribution to the wave energy resource (Contrib);

- The section in the middle presents the non-dimensional power performance parameters such as the non-dimensional performance of each zone $(\eta)$, its standard deviation $(s)$ and the corresponding confidence interval $(\mathrm{CI})$, which is based on the number of data points $(n)$ selected for the respective zone.

- The last section contains a combination of the prior two, as the average power production $(P)$, standard deviation and

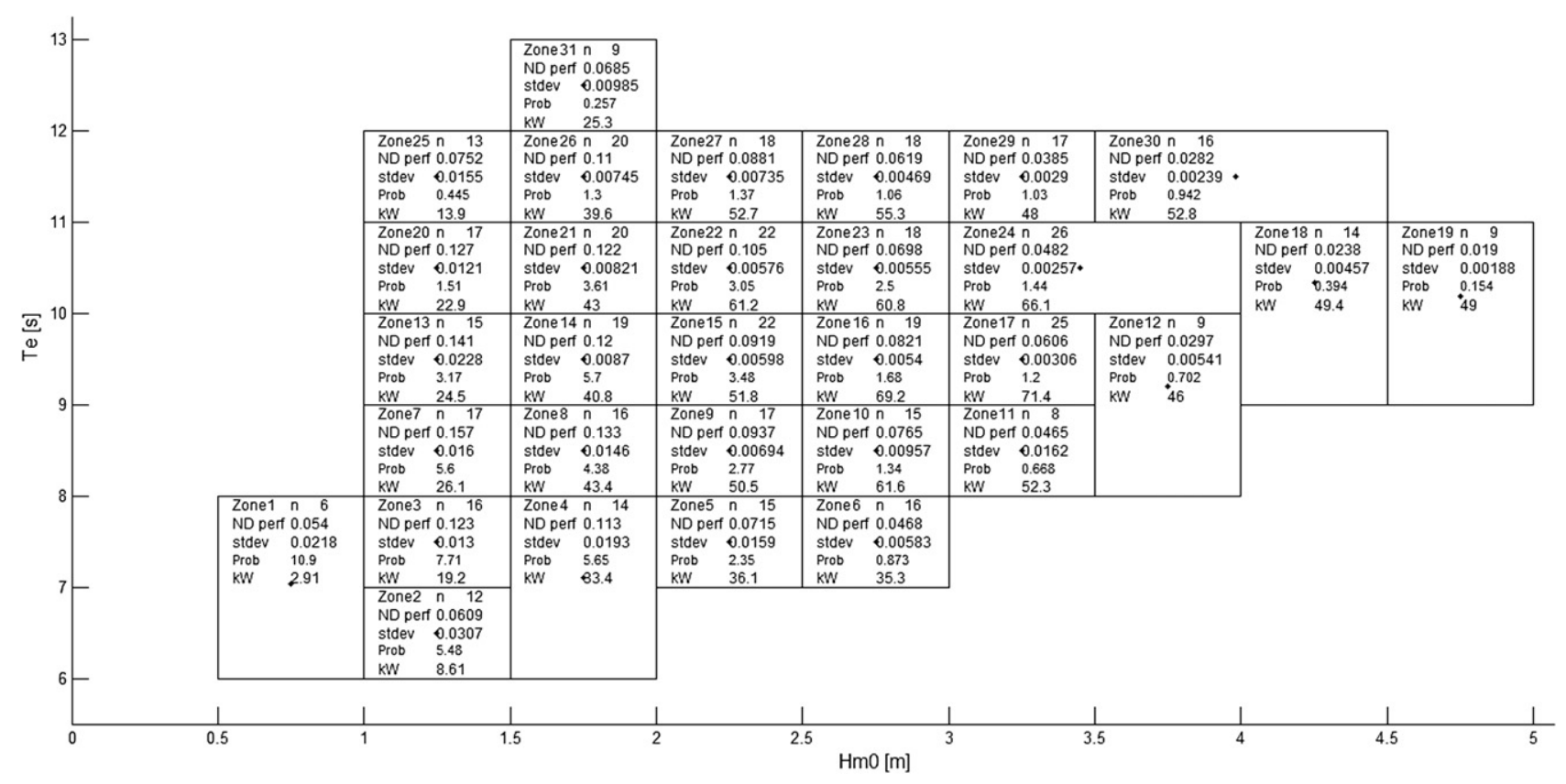

Fig. 5. Overview table containing some of the main environmental and performance parameters. 

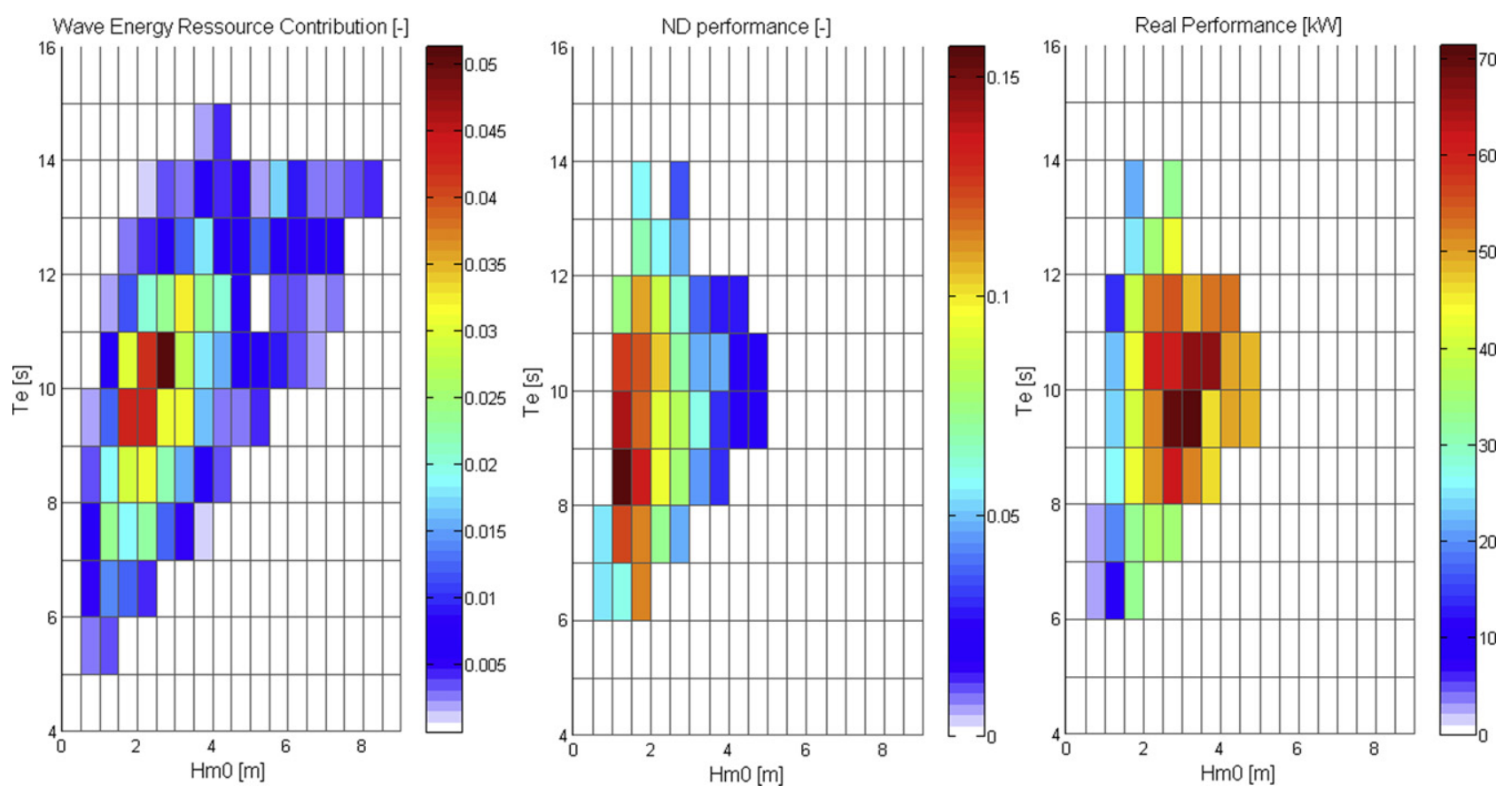

Fig. 6. Overview graphs of the wave energy contribution of each bin, and the corresponding non-dimensional performance and absolute power production.

confidence interval are calculated based on the nondimensional parameter and the corresponding available wave power.

The overall appraisal of environmental and performance values and the estimation of AEP and the load factor is given at the bottom of the Table 1 . Some of the values of the zones are simply summed, while others have to be calculated relative to a weighting function, which is relative to Contrib zone. $_{\text {. }}$

The overall non-dimensional performance $\left(\eta_{\text {overall }}\right)$ represents the weighted average of the non-dimensional performance of all the zones:

$\eta_{\text {overall }}=\sum_{\text {zone }=1}^{n} \eta_{\text {zone }} \cdot$ Contrib zone

An unbiased estimate of the overall standard deviation and confidence interval can be obtained by applying the following equation, in which $X$ can be replaced by $s$ or $\mathrm{CI}$ in order to obtain an overall value:
From the average power production of the WEC, the yearly total converted energy or annual energy production (AEP) can be calculated by multiplying the average power production by the number of hours in a year $(\approx 8766)$ :

AEP $=P_{\text {average }} \cdot 8766$

The load factor (LF) represents the average usage of the installed capacity:

$\mathrm{LF}=\frac{P_{\text {average }}}{P_{\text {installed }}}$

\subsection{Estimating the WEC performance at another location of interest}

The environmental matrix varies between different site locations and therefore a new environmental matrix (often reduced to the $H_{m 0}-T_{e}$ scatter diagram) should be created for each alternative location of interest.

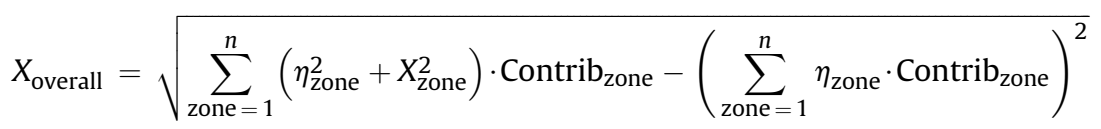

The average power production of the WEC can be obtained on the same way as the overall $\eta$, which corresponds to the sum of the power production of every zone, weighted with regards to its probability of occurrence (Eq. (5)). The corresponding uncertainty parameters can be obtained by multiplying their non-dimensional values by the average power production of the WEC:

$P_{\text {average }}=\sum_{\text {zone }=1}^{n} P_{\text {zone }} \cdot$ Prob $_{\text {zone }}$
If the device is intended to have a different size than in the original case, the original performance data of the zones requires scaling for the alternative location. The scaling ratio between the original and the new location of interest should be equivalent to the variation in size between the two physical models, e.g. if the original performance data were obtained on a 1:4 prototype deployed at a benign site and the performance is intended to be estimated for a full-scale WEC installed in the open ocean, then the environmental parameters that correspond to the non-dimensional 
Table 1

The summarising table of the WEC performance and its estimated energy production.

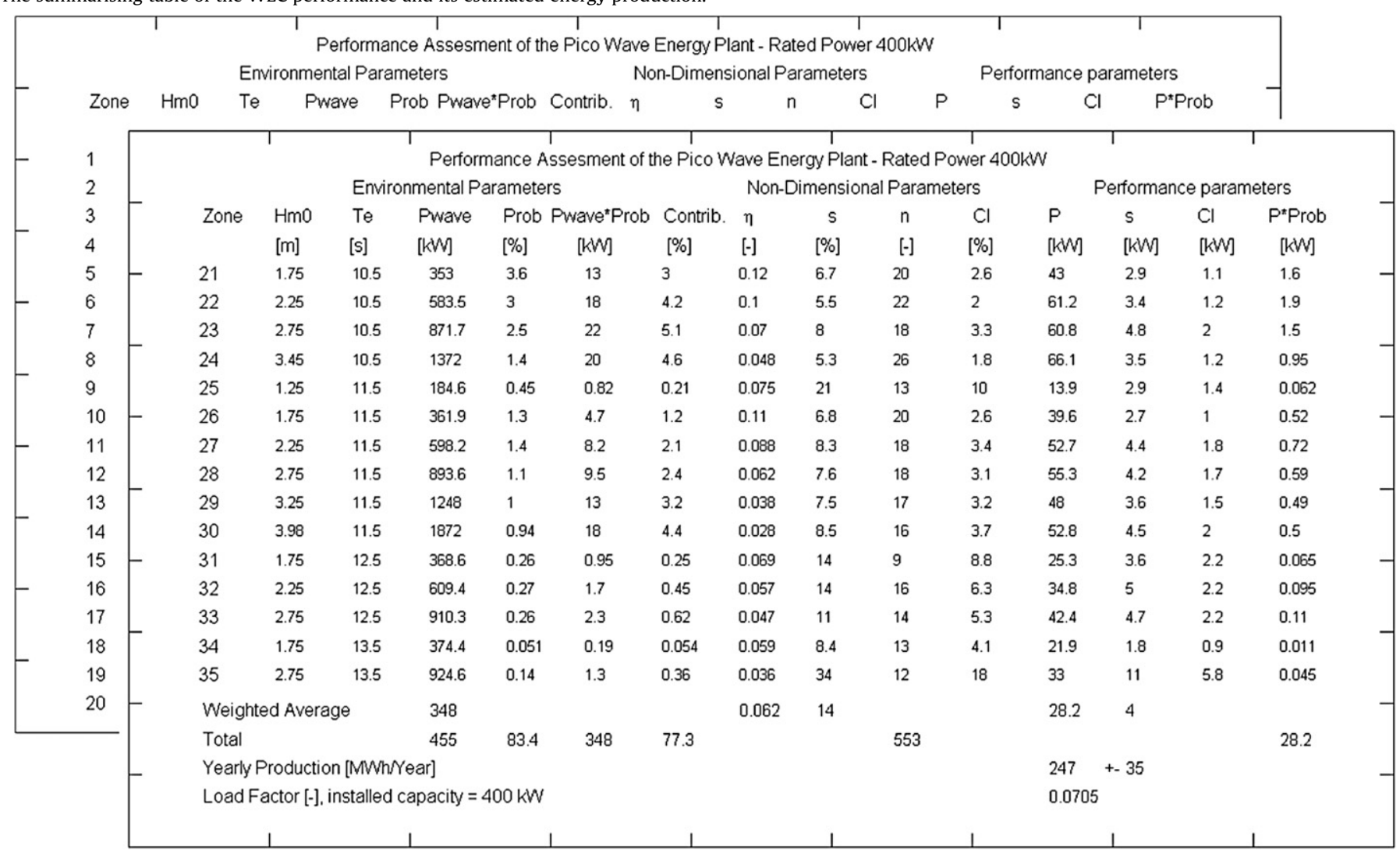

performance data should be scaled to the ratio of 1-4 following Froude's scaling law [14]. However, if the same scale model is to be used at another location, no scaling should be applied even if the wave energy content differs.

The losses that the device incurs throughout the power conversion chain (e.g. bearing and electrical losses) can change depending on the scale of the device. If the performance has been evaluated at different power conversion stages, these can be taken into account by adapting the $\eta$ of the corresponding power conversion stage.

The original definition of the zones can be adapted depending on how the scaled performance data overlaps the new environmental scatter diagram. Some zones might not cover any longer spaces where there is environmental or performance data and thereby become excessive, or others might need to be enlarged or created. Where parts of the new environmental matrix are not represented by a zone, then the performance in these zones might be calculated using a validated numerical tool (possibly validated against the original performance data).

Fig. 7 shows a schematic overview of the procedure. In the top left figure, performance data that were obtained using a reduced size model are presented on top of the wave energy contributions in the bins of the scatter diagram for the location of interest for the full-scale device. The performance data are then scaled to the environmental scatter diagram for the new location of interest in the top right figure.

This scaling ratio corresponds to the actual increase in size of the WEC, while maintaining geometrical similarity. In this figure, increasing colour darkness corresponds to a greater contribution to the wave energy resource of each bin. In the lower figures, rectangles mark zones that are initially defined where abundant performance data are available and ellipses are used to indicate blank but still relevant sea states. As long as the WEC is intended to operate in the corresponding conditions, the performance of these zones will need to be calculated through validated numerical tools. Obviously there is no need to estimate the performance of a WEC in e.g. $7 \mathrm{~m}$ waves if it is not intended to operate in them, and these areas on the environmental scatter diagram should just be left blank or the performance of the device should be set to zero for these conditions.

\subsection{Assessment of the WEC performance at various conversion steps}

The aim of this analysis is to create an overview of the various conversion steps. This is done by breaking the energy conversion chain from wave to wire into its various components and by presenting their behaviour over the environmental scatter diagram. As this creates a complete overview of the power conversion chain it can lead to a better understanding of the WEC, possibly giving valuable insights into the behaviour of some components of the device for specific environmental conditions.

Depending on the WEC and available data, the $\eta$ can be estimated at various conversion steps. After the data selection (which is stipulated in Section 4.2), the $\eta$ can then be calculated for the various conversion steps. It could be interesting to see if a different AEP would be obtained if the data selection procedure were based on another conversion step.

Fig. 8 presents as an example the conversion $\eta$ of an OWC plant from the wave power to pneumatic power in the chamber and then 

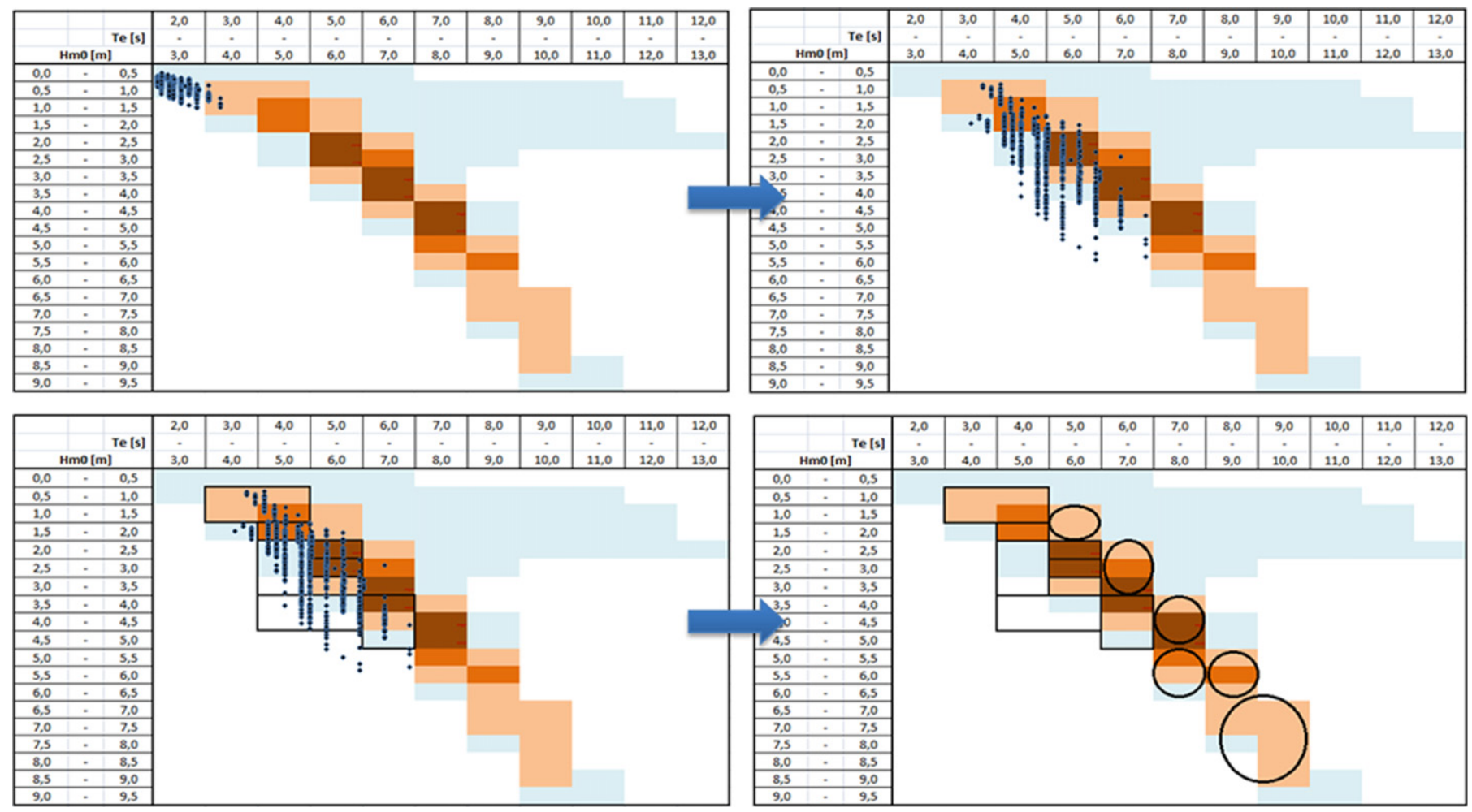

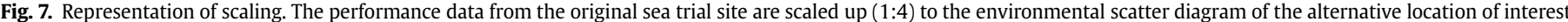

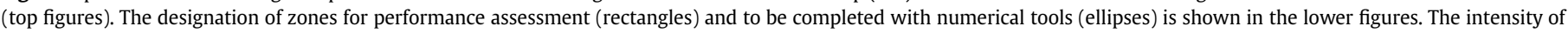
the background colour illustrates the level of the wave energy contribution (Contrib) of the bins.
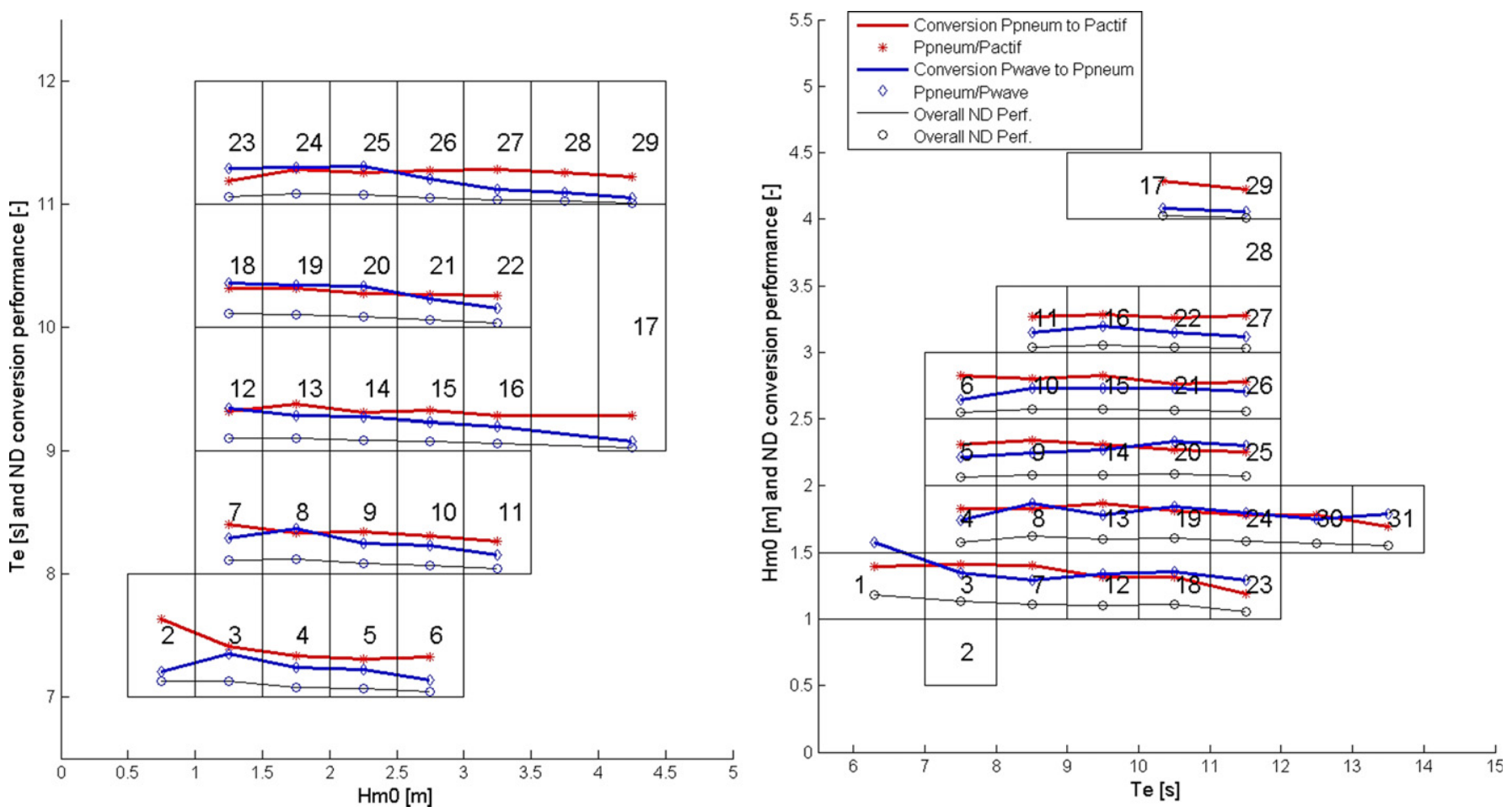

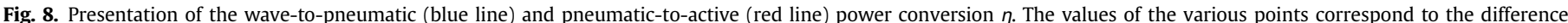

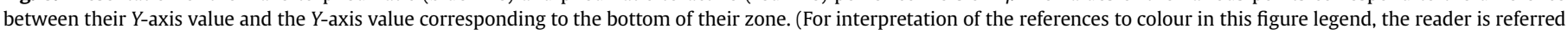
to the web version of this article.) 


\begin{tabular}{|c|c|c|c|c|c|c|c|c|c|c|c|c|c|c|c|}
\hline \multicolumn{15}{|c|}{ Performance from the sea trials performed at Point 3 in Danish North Sea - Directional spreading $=4-6$} & \\
\hline \multirow{3}{*}{ Zone } & \multicolumn{15}{|c|}{ Performance from the sea trials performed at Point 3 in Danish North Sea - Directional spreading $=2-4$} \\
\hline & \multirow{3}{*}{ Zone. } & \multicolumn{14}{|c|}{ Performance from the sea trials performed at Point 3 in Danish North Sea - Directional spreading $=0-2$} \\
\hline & & \multicolumn{6}{|c|}{ Environmental parameters } & \multicolumn{4}{|c|}{ Non-dimensional parameters } & \multicolumn{4}{|c|}{ Performance parameters } \\
\hline 1 & & & Hmo & $\mathrm{Te}$ & Pwave & Prob & Pwave.Prob & $\eta$ & s & $\mathrm{n}$ & $\mathrm{CI}$ & $P$ & s & $\mathrm{CI}$ & P.Prob \\
\hline 2 & 1 & Zone & {$[\mathrm{m}]$} & {$[\mathrm{s}]$} & {$[\mathrm{kW}]$} & {$[-]$} & {$[\mathrm{kW}]$} & {$[-]$} & {$[-]$} & {$[-]$} & {$[-]$} & {$[\mathrm{kW}]$} & {$[\mathrm{kW}]$} & {$[\mathrm{kW}]$} & {$[\mathrm{kW}]$} \\
\hline 3 & 2 & 1 & 1 & 5.6 & 59 & 0.468 & 55 & 0.195 & 0.041 & 80 & 0.009 & 12 & 2 & 1 & 5 \\
\hline 4 & 3 & 2 & 2 & 7.0 & 295 & 0.226 & 133 & 0.284 & 0.062 & 67 & 0.015 & 84 & 18 & 4 & 19 \\
\hline 5 & 4 & 3 & 3 & 8.4 & 797 & 0.108 & 172 & 0.152 & 0.044 & 48 & 0.013 & 121 & 35 & 10 & 13 \\
\hline 6 & 5 & 4 & 4 & 9.5 & 1603 & 0.051 & 164 & 0.098 & 0.029 & 13 & 0.017 & 157 & 46 & 28 & 8 \\
\hline \multirow[t]{4}{*}{ Weig } & 6 & 5 & 5 & 11.2 & 2953 & 0.024 & 142 & 0.063 & 0.015 & 27 & 0.006 & 186 & 44 & 17 & 4 \\
\hline & Weig & 6 & 6 & 13.0 & 4936 & 0.012 & 118 & 0.038 & 0.017 & 5 & 0.020 & 188 & 84 & 96 & 2 \\
\hline & & \multicolumn{3}{|c|}{ Weighted average } & 392 & & & 0.133 & 0.069 & & 0.027 & 52 & 27.0 & 10.6 & \\
\hline & & \multicolumn{3}{|c|}{ Total } & & 0.889 & 392 & & & & & & & & 52 \\
\hline
\end{tabular}

Fig. 9. Performance results of the various data sets following a third influential parameter (directional spreading).

from this pneumatic power to the active (electrical) power output, over the various zones. It is structured in a way that the curves cross through zones that have an identical environmental parameter $\left(H_{m 0}\right.$ or $T_{e}$ ). The curves follow the same axis as the environmental parameters, except that their reference must be translated to the bottom of their respective zone.
5.4. Assessment of the influence of a specific environmental or device dependent parameters

This analysis can give an insight on the influence of other environmental or device dependent parameters - besides $H_{m 0}$ and $T_{e}-$ on the overall performance or specific power conversion steps.

\begin{tabular}{|c|c|c|c|c|c|c|c|c|c|c|c|c|c|c|}
\hline \multicolumn{15}{|c|}{ Performance from the sea trials performed at Point 3 in Danish North Sea } \\
\hline \multicolumn{7}{|c|}{ Environmental parameters } & \multicolumn{4}{|c|}{ Non-dimensional parameters } & \multicolumn{4}{|c|}{ Performance parameters } \\
\hline Zone & $\begin{array}{l}\text { Hmo } \\
{[\mathrm{m}]}\end{array}$ & $\begin{array}{l}\mathrm{Te} \\
{[\mathrm{s}]}\end{array}$ & $\begin{array}{c}\text { Direct. } \\
\text { Spreading }\end{array}$ & $\begin{array}{l}\text { Pwave } \\
{[\mathrm{kW}]}\end{array}$ & $\begin{array}{c}\text { Prob } \\
{[-]}\end{array}$ & $\begin{array}{c}\text { Pwave.Prob } \\
{[\mathrm{kW}]}\end{array}$ & $\eta$ & $\begin{array}{c}s \\
{[-]}\end{array}$ & $\begin{array}{c}\mathrm{n} \\
{[-]}\end{array}$ & $\begin{array}{l}\text { CI } \\
{[-]}\end{array}$ & $\begin{array}{c}\mathrm{P} \\
{[\mathrm{kW}]}\end{array}$ & $\begin{array}{c}\mathrm{s} \\
{[\mathrm{kW}]}\end{array}$ & $\begin{array}{c}\mathrm{CI} \\
{[\mathrm{kW}]}\end{array}$ & $\begin{array}{l}\text { P.Prob } \\
{[\mathrm{kW}]}\end{array}$ \\
\hline \multirow[t]{3}{*}{1} & 1 & 5.6 & 1.0 & 59 & 0.198 & 12 & 0.195 & 0.010 & 12 & 0.029 & 12 & 0.6 & 1.7 & 2 \\
\hline & & & 3.0 & 59 & 0.160 & 9 & 0.155 & 0.008 & 8 & 0.018 & 9 & 0.5 & 1.1 & 1 \\
\hline & & & 5.0 & 59 & 0.110 & 6 & 0.115 & 0.006 & 6 & 0.010 & 7 & 0.3 & 0.6 & 1 \\
\hline \multirow[t]{3}{*}{2} & 2 & 7.0 & 1.0 & 295 & 0.110 & 32 & 0.284 & 0.014 & 15 & 0.060 & 84 & 4.2 & 17.9 & 9 \\
\hline & & & 3.0 & 295 & 0.066 & 19 & 0.179 & 0.009 & 6 & 0.024 & 53 & 2.6 & 7.1 & 3 \\
\hline & & & 5.0 & 295 & 0.050 & 15 & 0.152 & 0.008 & 7 & 0.017 & 45 & 2.2 & 5.1 & 2 \\
\hline \multirow[t]{3}{*}{3} & 3 & 8.4 & 1.0 & 797 & 0.048 & 38 & 0.152 & 0.008 & 14 & 0.017 & 121 & 6.1 & 13.8 & 6 \\
\hline & & & 3.0 & 797 & 0.035 & 28 & 0.124 & 0.006 & 7 & 0.012 & 99 & 4.9 & 9.2 & 3 \\
\hline & & & 5.0 & 797 & 0.025 & 20 & 0.096 & 0.005 & 5 & 0.007 & 77 & 3.8 & 5.5 & 2 \\
\hline \multirow[t]{3}{*}{4} & 4 & 9.5 & 1.0 & 1603 & 0.021 & 34 & 0.098 & 0.005 & 7 & 0.007 & 157 & 7.9 & 11.5 & 3 \\
\hline & & & 3.0 & 1603 & 0.017 & 27 & 0.072 & 0.004 & 6 & 0.004 & 115 & 5.8 & 6.2 & 2 \\
\hline & & & 5.0 & 1603 & 0.013 & 21 & 0.051 & 0.003 & 5 & 0.002 & 82 & 4.1 & 3.1 & 1 \\
\hline \multirow[t]{3}{*}{5} & 5 & 11.2 & 1.0 & 2953 & 0.012 & 35 & 0.063 & 0.003 & 6 & 0.003 & 186 & 9.3 & 8.8 & 2 \\
\hline & & & 3.0 & 2953 & 0.007 & 21 & 0.038 & 0.002 & 5 & 0.001 & 112 & 5.6 & 3.2 & 1 \\
\hline & & & 5.0 & 2953 & 0.005 & 15 & 0.012 & 0.001 & 5 & 0.000 & 35 & 1.8 & 0.3 & 0 \\
\hline \multirow[t]{3}{*}{6} & 6 & 13.0 & 1.0 & 4937 & 0.009 & 44 & 0.038 & 0.002 & 5 & 0.001 & 188 & 9.4 & 5.3 & 2 \\
\hline & & & 3.0 & 4937 & - & - & - & - & - & - & - & - & - & - \\
\hline & & & 5.0 & 4937 & - & - & - & - & - & - & - & - & - & - \\
\hline \multicolumn{3}{|c|}{ Weighted average } & & 378 & & & 0.133 & 0.006 & & 0.013 & 42 & 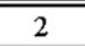 & $\overline{5}$ & \\
\hline & Total & & & & 0.886 & 378 & & & & & & & & 42 \\
\hline \multicolumn{11}{|c|}{ Yearly Production $[\mathrm{MWh} / \mathrm{y}]$} & 367 & & & \\
\hline \multicolumn{11}{|c|}{ Load factor [-] (400kW installed capacity) } & 0.10 & & & \\
\hline
\end{tabular}

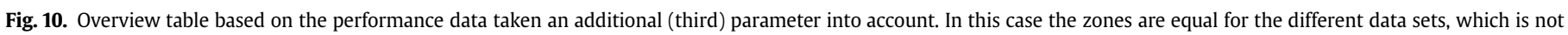
mandatory. (This figure is based on illustrative values). 
It can also provide a more accurate estimation of the AEP, resulting from the enhanced resolution of the environmental climate. As long as it is configurable and measurable, any device dependent parameter - either physical or logical (e.g. control strategy) - can be considered.

At the beginning of the procedure, the original performance data should be grouped following the new parameter of interest into separate data sets. The same data selection procedure can be applied on each individual data set, resulting in n-dimensional matrices. This is represented in Fig. 9 by various tables containing the performance and the related uncertainty for the different zones.

Although the zones do not need to be the same for the various data sets, this facilitates the performance comparison. Based on the individual results of all the zones, an overview table can be made estimating the overall performance and AEP. This table can thus reveal more detail than the one based on only 2 influential parameters (Table 1). An example of this is given in Fig. 10. If the environmental matrix of another location of interest includes information concerning this third parameter, then a more accurate estimation of the AEP can also be made for this new location.

In order to have a better global schematic representation of the influence of a third parameter, a similar graph can be drawn as Fig. 8. This will demonstrate the trend and variation of the performance due to the third parameter of influence for different wave heights or wave periods, holding one of them constant.

\section{Conclusions and further development}

The presented methodology provides an equitable means to estimate the power production capabilities of a WEC based on trials within the natural environment, where incident environmental conditions are not prescribed. The methodology includes an appreciation of the uncertainties associated with the estimated performance. Several approaches for presenting the mean performance are suggested. It has the benefits of being fully based on performance data from sea trials (i.e. realistic uncontrollable conditions) of the WEC and also that it presents the accuracy of the stated performance. The developer has the opportunity to use a common but adaptable approach that allows a selection of the performance data in a transparent way, which does not punish the "trial and error" testing and that rewards the persistent demonstration of the capabilities of the device.

The estimation of the power production capabilities can be expressed in terms of annual energy production at the test location or any other location of interest, however it can also include input from various performance data analysing and presentation tools. By considering other environmental parameters than the predominant $H_{m 0}$ and $T_{e}$, the environmental conditions can be described with a higher resolution. An identical procedure can be followed in order to analyse the influence of configurable elements of the WEC, e.g. control laws or the adaption of physical components.

The power conversion chain, from wave power to generated electricity, can also be investigated following the same principle. It enables a developer to observe, both graphically and numerically, the variations of the performance at various conversion steps for various sea states, and facilitates the creation of an overview of the behaviour of the WEC.

In practice, the same methodology can also be applied to other energy converting technologies, such as tidal energy converters [9]. The presentation of the methodology was based on wave energy but the environmental matrix can be adapted in order to suit other technologies, which also have their own power conversion chain.

The work leading to this paper has by large been carried out within the EU-FP7 funded project EquiMar, which ended in spring 2011. The key outcome of the EquiMar project is presented in [8] and [9]. International efforts to develop guidelines and standards within the field of marine renewable energies also include the IEC TC-114. Within TC-114 a new work item, to follow up on [15], has been established by the Danish Standardization Organization based on the methodology presented in this paper. Thus, it is the intention to develop this methodology into a Technical Specification within the IEC framework. The methodology has already been applied on two different WECs, the results of which are given in [12] and [13].

\section{Acknowledgements}

The authors gratefully acknowledge the financial support from the EU Commission for the EquiMar project (Grant Agreement No. 213380), as well the Marie Curie Initial Training Network WaveTrain2 project (Grant Agreement No. 215414) and the Danish Council for Strategic Research under the Programme Commission on Sustainable Energy and Environment (Contract 09-067257, Structural Design of Wave Energy Devices) which made this work possible.

\section{References}

[1] Griffiths JW. Benefits of standards development for the ocean energy industry. In: Proceedings of the 9th European Wave and Tidal Energy Conference (EWTEC); 2011.

[2] Nadeau M. Developing international standards for marine energy converters In: Proceedings of the 3rd International Conference and Exhibition on Ocean Energy (ICOE); 2010.

[3] Holmes B, Nielsen K. Task 2.1 guidelines for the development \& testing of wave energy systems. OES IA Document No T02-2.1; 2010.

[4] Smith G, Taylor J. Preliminary wave energy device performance protocol. The Department of Trade and Industry (DTI); 2007. MRF/02/00005/00/00URN 07/807.

[5] Pitt E. Assessment of performance of wave energy conversion systems. The European Marine Energy Centre Ltd (EMEC); 2009. no. Marine Energy Guides, p. $1-28$.

[6] Nielsen K. Development of recommended practices for testing and evaluating ocean energy systems. OES IA Annex 2-Document No T02-0.0; 2010.

[7] Hagerman G, Bedard R. Specification guidelines for preliminary estimation of power production by offshore wave energy conversion devices. EPRI; 2003. p. $1-16$.

[8] Ingram D, Smith G, Bittencourt-Ferreira C, Smith H. Protocols for the equitable assessment of marine energy converters. University of Edinburgh, ISBN 978-09508920-2-3; 2011.

[9] Kofoed JP, Pecher A, Margheritini L, Holmes B, McCombes T, Johnstone C, et al. Data analysis \& presentation to quantify uncertainty, equiMar deliverable D4.2; 2010.

[10] TC114/TS62600-101 Committee Draft (CD). Marine energy - wave, tidal and other water current converters - part 101: wave energy resource characterization and assessment. IEC; 2011.

[11] Smith G, Taylor T. Preliminary wave energy device performance protocol, final technical report. OES-IA document no T02-T03.1, 2007.

[12] Pecher A, Le Crom I, Kofoed JP, Neumann F, de Brito Azevedo E. Performance assessment of the Pico OWC power plant following the equiMar methodology. In: Proceedings of the twenty-first International Offshore and Polar Engineering Conference (ISOPE), TPC-447; 2011.

[13] Parmeggiani S, Chozas JF, Pecher A, Friis-Madsen E, Sørensen HC, Kofoed JP. Performance assessment of the Wave Dragon WEC based on the equiMar methodology. In: Proceedings of the 9th European Wave and Tidal Energy Conference (EWTEC); 2011.

[14] Kofoed JP, Frigaard P. Development of wave energy converters: the Danish case. Renewable Ocean Energy 2009;4(4):83-96.

[15] TC114/TS62600-100 Draft Technical specifications. Marine energy - wave tidal and other water current converters - part 100: power performance assessment of electricity producing wave energy converters. IEC; 2011. 